\title{
Phronesis
}

\section{La formation des bénévoles : une démarche de professionnalisation?}

Analyse de la mise oeuvre des dispositifs d'accompagnement et de formation des bénévoles dans trois centres sociaux

\section{parisiens}

\section{Volunteer training: a process of professionalisation?}

Analysis of the implementation of the devices for the support

\section{Florence Tardif Bourgoin}

\section{Volume 2, numéro 4, octobre 2013}

Du bon usage de la notion de "professionnalisation " : entre modèle de formation, élaborations des politiques et attentes des acteurs; quelles convergences?

URI : https://id.erudit.org/iderudit/1022261ar

DOI : https://doi.org/10.7202/1022261ar

Aller au sommaire du numéro

Éditeur(s)

Institut de recherche sur les pratiques éducatives

ISSN

1925-4873 (numérique)

Découvrir la revue

Citer cet article

Tardif Bourgoin, F. (2013). La formation des bénévoles : une démarche de professionnalisation? Analyse de la mise oeuvre des dispositifs d'accompagnement et de formation des bénévoles dans trois centres sociaux parisiens. Phronesis, 2(4), 61-69. https://doi.org/10.7202/1022261ar

\section{Résumé de l'article}

Les exigences de professionnalisation dans le champ de l'action sociale préoccupent les associations dans un contexte de réduction des financements. Les référentiels de compétences accompagnent ainsi le recrutement des professionnels et les tendances à la sélection des bénévoles ainsi que la formulation d'objectifs en vue de leur qualification. Dans un contexte d'éducation populaire (par exemple dans les centres sociaux) favorisant la transmission des savoirs et des expériences dans un objectif de construction partagée, quelles places occupent la formation et la professionnalisation des bénévoles? d'utilisation que vous pouvez consulter en ligne.

https://apropos.erudit.org/fr/usagers/politique-dutilisation/ 


\title{
La formation des bénévoles : une démarche de professionnalisation? Analyse de la mise œuvre des dispositifs d'accompagnement et de formation des bénévoles dans trois centres sociaux parisiens
}

\author{
Florence TARDIF BOURGOIN
}

Laboratoire CRF - CNAM Paris

IRTS Paris 145 avenue parmentier

75010 Paris

Florence.bourgoin@irts.paris.idf.asso.fr

Mots clés : bénévolat, centre social, compétences, cultures de formation, développement professionnel et personnel, professionnalisation.

Résumé : Les exigences de professionnalisation dans le champ de laction sociale préoccupent les associations dans un contexte de réduction des financements. Les référentiels de compétences accompagnent ainsi le recrutement des professionnels et les tendances à la sélection des bénévoles ainsi que la formulation dobjectifs en vue de leur qualification. Dans un contexte déducation populaire (par exemple dans les centres sociaux) favorisant la transmission des savoirs et des expériences dans un objectif de construction partagée, quelles places occupent la formation et la professionnalisation des bénévoles?

Title : Volunteer training: a process of professionalisation? Analysis of the implementation of the devices for the support and training of volunteer in three Parisian social centers

Key-words : volunteering, social center, skills training cultures, professional and personal development, professionalization.

Abstract: The professionalization requirement in the field of social action worries associations in the financing decreasing context. Targets within the sight of volunteers skills are expressed. In a context of people's education (social centers) promoting knowledge and practical experiences transmission in a goal of shared construction, what is the position occupied by volunteers training and professionalization? 
Face à la professionnalisation de l'action sociale puis l'arrivée des professionnels de l'animation et du développement local, les bénévoles des centres sociaux se sont progressivement associés à des champs divers de compétences permettant de mettre en ouvre l'Animation globale. La formation des bénévoles est depuis longtemps (années 60) une priorité ancrée dans une démarche participative visant la promotion sociale individuelle et collective (démarche déducation populaire). Aujourd'hui, face aux enjeux de professionnalisation qui accompagnent la qualité des interventions sociales (loi 2002-2), les projets des structures séquilibrent constamment entre d'une part la formation et la qualification des acteurs salariés et bénévoles et d’autre part une démarche d’ouverture prônant la diversité des expériences (pour contrebalancer les exigences des financeurs du côté des compétences).

Après une présentation du contexte démergence de la recherche et des questionnements associés (I-1), l'identification du cadre théorique de référence retenu (I-2) et les méthodes d’enquête utilisées (I-3), nous proposons dans cet article, à partir du matériau recueilli, d’opérer une analyse des résultats selon deux orientations : regarder les analogies repérées entre les dispositifs classiques de professionnalisation et les cultures de formation déployées en centre social (II-1) et comprendre la place du développement personnel dans ces dispositifs de professionnalisation (II-2). Il s’agira enfin d'identifier les enjeux sociaux qui se dégagent de ces pratiques en émergence, que ce soit du côté des logiques institutionnelles (III-1) ou individuelles (III-2) de professionnalisation. Pour conclure, les enjeux qui se développent autour du tutorat seront analysés au regard de ce qu'ils impliquent dans un contexte déducation populaire (promotion sociale et développement personnel associé).

\section{Lorigine d’une problématique de recherche sur la professionnalisation du bénévolat}

\subsection{Contexte et questionnements}

Les centres sociaux sont des associations dont les financements sarticulent à l'action publique (Tchernonog, 2008). et leurs missions se déclinent principalement dans des actions visant l'insertion, le lien social et la citoyenneté. Or, la question de la qualification des bénévoles cherche parfois à répondre à des exigences en lien avec le renouvellement du contrat $\mathrm{CNAF}^{1}$. Les financeurs attendent même parfois que les bénévoles soient formés. Ainsi les centres sociaux ont développé une formalisation de l'engagement bénévole qui permet de donner un cadre aux questions de formation et de professionnalisation des bénévoles. Les fédérations formulent des objectifs en vue de la qualification des bénévoles et l'acquisition de compétences via la formation peut alors faciliter une démarche d'insertion professionnelle. Mais la formation des bénévoles vise aussi l'appropriation d'une démarche collective et de l'esprit centre social qui doit encourager la transmission des valeurs de léducation populaire. Le bénévole qui s’engage porte le message et les valeurs du centre : autonomie des publics, participation, égalité.... Sa libre adhésion s'accompagne donc d'une acceptation d'un certain nombre de lignes directrices qui vont accompagner sa démarche. Mais si la formation professionnelle vise l'acquisition des compétences en lien avec une activité donnée, léducation populaire prône plus largement la promotion sociale des individus et leur participation à la vie citoyenne.

\subsection{Problématique de recherche et cadre théorique de référence}

Larticulation formation/professionnalisation est donc au cœur du questionnement retenu : Comment les centres sociaux accompagnentils la professionnalisation de leurs bénévoles au regard des valeurs déducation populaire qui fondent lorigine de leur démarche? La professionnalisation, au moment de poser cette question de départ est entendue dans trois dimensions complémentaires : l'ajustement des compétences professionnelles aux référentiels de l'action sociale (logique politique), les attentes des associations en matière de recrutement (logique institutionnelle) et le processus de qualification attendue en vue d'une insertion professionnelle. La question du projet social de l'organisation à l'origine de l'intention de professionnalisation se pose alors: Dans des organisations [...] affirmant un projet social, il est parfois proposé d’associer développement professionnel et développement personnel. Du développement professionnel au développement personnel (Wittorski, 2007), l'enjeu est alors d'identifier la place laissée au développement personnel et culturel dans une offre de professionnalisation qui s’appuie sur des dispositifs qui cherchent d’abord à développer des compétences finalisées dans des situations de travail.

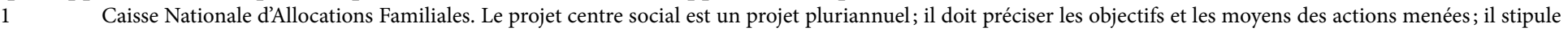

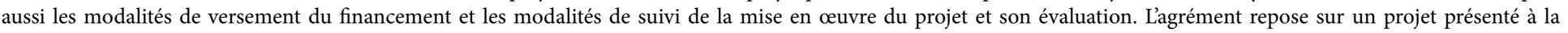

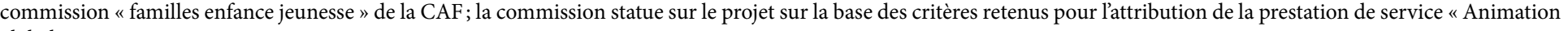
globale». 
Le concept central de la recherche s'organise autour des logiques de professionnalisation à partir de la conceptualisation de Wittorski (2007). En distinguant la professionnalisation des activités et des structures (logiques institutionnelles) de celle des individus (attentes individuelles), ce cadre de référence permet de faire émerger des analogies avec les pratiques d’accompagnement des bénévoles (cultures de formation associées). Cette approche permet d'associer les logiques d'acteurs aux enjeux associatifs (gouvernance, reconnaissance, participation et promotion sociale), notamment autour de la question des valeurs : "L'exigence démocratique dévaluation, dont les déclinaisons en termes de qualité sont l'un des derniers avatars, se fonde sur le principe que la valeur d'une démarche doit être appréciée non seulement à l'aune des idéaux auxquels elle se réfère, des résultats effectifs, mais aussi de son coût, de son rendement, et plus généralement de son efficience, dans une logique comparative ». (Lesain-Delabarre, 2006, p. 619)

La problématique finalement retenue s'est organisée autour des influences des valeurs déducation populaire sur les pratiques de professionnalisation des centres sociaux à l'égard des bénévoles. Les modes de gouvernance associative, en s'inscrivant dans une logique de transmission des valeurs qui doit s'articuler avec les logiques de professionnalisation, engagent les structures centre social dans le développement de cultures de formation qui interrogent la place des bénévoles dans un projet participatif déducation populaire (développement personnel/ développement professionnel).

\subsection{Modalités d'enquête}

La recherche s'est appuyée sur le choix de trois structures parisiennes. Un premier rencontré en phase exploratoire (en parallèle d'un entretien mené auprès de la fédération parisienne concernée) a été conservé pour la suite de l'enquête (centre 1). Pour les autres centres, une structure a été retenue dans le réseau des auditeurs (qui disposait d'une équipe de bénévoles assez nombreuse et diversifiée : centre 2). Le dernier centre (centre 3) a été choisi sur la base de sa participation à un document publié par la fédération parisienne concernant la participation des bénévoles et l'accès aux formations dans ce contexte ${ }^{2}$. Cibler une activité en centre social ${ }^{3}$ a ensuite été nécessaire, car il en découle des exigences de professionnalisation différentes selon le secteur d'intervention. Le choix de l'accueil est alors apparu comme une entrée intéressante; l'accueil social étant propice à la diffusion des valeurs d’éducation populaire sur un territoire (relais des informations et des services présents sur le quartier).

Le recueil des données s'est inscrit dans une démarche méthodologique d'entretiens semi-directifs : 2 entretiens auprès de Directeurs (centres 1 et 2) et 2 entretiens auprès de référents de l'activité d'accueil (centre 1 et 3). Cette première phase d'investigation devait permettre le repérage et l'analyse des enjeux inhérents au déploiement de la professionnalisation organisationnelle (et des cultures de formation qui en découlent) permettant une compréhension de la mise en œuvre des dispositifs : contextes de mise en œuvre des voies de professionnalisation en centre social, impact sur les compétences attendues du côté des bénévoles, place et enjeux de l’accompagnement individuel. La préparation du protocole d'enquête s'est appuyée sur l'identification préalable d’objectifs spécifiques pour chaque catégorie d'acteurs; permettant de construire ensuite les guides d'entretien correspondants.

Auprès des Directeurs :

- Comprendre les enjeux institutionnels de professionnalisation à léchelle du centre social;

- Identifier l'articulation entre les valeurs d’éducation populaire et la professionnalisation;

- Mesurer les effets sur l’accueil et l’accompagnement des bénévoles.

Auprès des référents des bénévoles/coordinateurs de l’activité accueil :

- Identifier le degré de formalisation de l’activité accueil, les attentes vis-à-vis des bénévoles;

- Analyser les modalités et représentations de la fonction d’accompagnement;

- Repérer la place de léducation populaire dans les pratiques.

$2 \quad$ Les bénévoles dans les centres sociaux de Paris : de l'accueil à la formation - Sept 2003.

3 En retenant la dimension de l'accueil social aux côtés d'autres délimitations possibles de lobjet de recherche (telles qu’une catégorie de bénévoles ou un territoire précis); cela m’a permis de conserver la diversité des profils bénévoles dans une logique de complémentarité au service d'une même mission et de mettre en valeur l'importance de lancrage territorial (accueil dans sa dimension de développement local) dans la logique d'animation globale. 
Tableau 1:

Grilles d’analyse des entretiens

\begin{tabular}{|l|l|l|}
\hline $\begin{array}{l}\text { Dimensions de la } \\
\text { professionnalisation }\end{array}$ & Attentes vis-à-vis des bénévoles & Valeurs d’éducation populaire associées \\
\hline $\begin{array}{l}\text { Structure centre } \\
\text { social : degré de } \\
\text { professionnalisation } \\
\text { (recrutement, diplômes) }\end{array}$ & $\begin{array}{l}\text { Intégration dans une culture } \\
\text { institutionnelle associant des compétences } \\
\text { et des valeurs }\end{array}$ & $\begin{array}{l}\text { Diffusion du projet associatif et adhésion } \\
\text { aux valeurs du centre//fédération? }\end{array}$ \\
\hline $\begin{array}{l}\text { Activité Accueil social: } \\
\text { degré de formalisation de } \\
\text { lactivité (fiches de poste...) }\end{array}$ & $\begin{array}{l}\text { Attentes en termes de pratiques } \\
\text { professionnelles (savoirs, savoirs-être) }\end{array}$ & $\begin{array}{l}\text { Respect des valeurs d’éducation populaire } \\
\text { dans l'accueil des publics }\end{array}$ \\
\hline $\begin{array}{l}\text { Bénévole : pratiques } \\
\text { d'accompagnement } \\
\text { (individuel/collectif, outils, } \\
\text { tutorat...) }\end{array}$ & $\begin{array}{l}\text { Motivations et parcours individuels en lien } \\
\text { avec les enjeux de l'activité et de la structure }\end{array}$ & $\begin{array}{l}\text { Enjeu de promotion sociale individuelle et } \\
\text { collective }\end{array}$ \\
\hline
\end{tabular}

\section{Analyse des résultats : des voies de professionnalisation au développement personnel et professionnel}

\subsection{Cultures de formation en centre social et voies de professionnalisation associées}

Dans les trois structures investiguées, quatre profils d’accompagnement/formation (cultures de formation) se sont détachés; ils mettent en jeu des modes d’adhésion différents aux valeurs de léducation populaire qui influencent la place des bénévoles :

- La figure du tuteur : il s’agit de l'accompagnement individuel des bénévoles à travers' une phase d'intégration qui s’apparente à une forme de tutorat (repérage des missions, transmission des outils techniques, mise à disposition des moyens matériels) et qui vise la prise en charge de l'activité par le bénévole;

- La figure du collègue : Des temps de réunions ou de participation constituent des moments conviviaux qui permettent d'assurer l'intégration du bénévole dans léquipe et la compréhension plus globale du contexte dans lequel la structure se positionne;

- La figure du formateur : Des formations techniques en interne visent l'appropriation d'outils spécifiques en lien avec l'activité (gestion de laccueil, scolaire, écrivain public...);

- La figure de l'animateur : Des formations fédérales visent la compréhension de l'activité dans son contexte de centre social ou d'analyse des pratiques (temps de réflexion et de questionnements regroupant les participants de plusieurs centres).

\section{Nous allons voir ces quatre profils de manière un peu plus approfondie :}

\subsubsection{L’accompagnement individuel des bénévoles : la figure du tuteur ${ }^{4}$}

- On prend le temps qu'il faut; la formation c’est surtout de laccompagnement individuel entre le référent et le bénévole.

Le référent d'activité consacre du temps à la formation du bénévole qui arrive

- on lui donne les clés de la compréhension de la démarche

il attend en retour que le bénévole respecte le public en évitant de laisser tomber un groupe avec lequel une relation de confiance sest établie. La régularité consacrée au bénévolat, inscrite dans la charte d’engagement, permet de maintenir les projets alors même que le bénévole n’est pas sous contrat de subordination et reste libre de partir quand il veut.

\section{Un temps d'adaptation}

Dans le centre 1 : Il y a eu un début, on ne va pas dire de formation, mais une petite formation pour lui expliquer; quelle visualise les travailleurs sociaux, les appels téléphoniques, à qui ils s’adressent... Puis après la connaissance des activités, le yoga... les lieux, les gens... elle était avec nous à l'accueil et elle prenait des notes. Elle nous expliquait quand elle n’avait pas compris. Et puis c’est venu tout seul, maintenant elle se débrouille toute seule. De toute façon, que l'on soit salarié ou bénévole, il y a un temps d'adaptation ${ }^{\circledR}$.

Dans le centre 3 : Après le premier entretien, on fait un petit temps dessai en situation, cela permet à chacun de savoir si cela va correspondre aux attentes, autant du côté du professionnel que du bénévole. C'est un temps d’observation qui va permettre d’acquérir les compétences nécessaires à la réalisation de l'activité en autonomie... pour s'assurer que l'autre a compris, qu'il a toujours envie... beaucoup d'échanges.

4 Selon R. Wittorski, la voie $\mathrm{n}^{\circ} 5$ de professionnalisation est la logique de traduction culturelle par rapport à laction; la « figure » correspondante est celle du tuteur. $5 \quad$ Entretien Référent accueil centre 3. 


\section{La transmission des outils}

Dans le centre 3, la priorité pour le référent est de former les bénévoles de l'accueil aux outils; cette formation passe d’abord par un accompagnement individuel. Déjà une formation aux outils d'accueil; et puis ce qu'est un accueil en centre social'.

Cette transmission occupe une place prépondérante dans l'accompagnement des bénévoles; la maîtrise d'Excel est un critère de sélection.

Dans le centre 2, pour éviter le désengagement des bénévoles dans la gestion de l'outil statistique, la mise en place d'un cahier d’accueil a permis de trouver un compromis.

\section{Les conditions matérielles}

Au-delà des outils d'accompagnement développés (annonce Espace bénévolat, entretien, signature de la charte d’engagement, formation), le référent d'activité a un rôle à jouer dans l'aide qu'il met en place pour que le bénévole puisse réaliser son activité dans de bonnes conditions; il doit mettre à la disposition du bénévole les moyens matériels nécessaires au bon déroulement de la séance.

- Le professionnel il est là pour faire en sorte que son activité se déroule le mieux possible... le bénévole, il doit trouver sa table, il doit trouver ses chaises, il doit trouver ses feutres?.

La transmission des valeurs

La transmission des valeurs est réalisée de façon différente selon les structures. Elle occupe une place prépondérante dans le centre 3 (le projet associatif, qui a déjà fait l'objet d'une présentation au moment de l'entretien de recrutement, est repris par le référent d'activité avec le bénévole pour lui permettre de se repérer dans ses missions). Dans le centre 2, elle est seulement soumise aux questionnements des bénévoles desquels on attend une certaine curiosité. Finalement, dans le centre 1, la question des valeurs est abordée au moment de l'entretien (par le directeur) et fait l’objet de la charte d’engagement; mais le salarié référent n’y revient pas forcément dans la suite de l'accompagnement.

Ces pratiques d'accompagnement s'inscrivent dans la voie ${ }^{\circ} 5$ de professionnalisation que Wittorski (2007) nomme « logique de traduction culturelle par rapport à l'action » (p. 119) : «Les situations de travail où un tiers accompagne des salariés dans la réalisation d'une activité. Ce tiers assure une fonction de transmission de savoirs ou de connaissances, mais aussi une fonction de mise à distance de l'action, des modifications des façons de voir et de penser l'action... tutorat, parrainage, accompagnement, conseil » (Ibid.). Ces pratiques sont communes aux trois centres : L’accueil du bénévole (par le référent qui devient le tuteur) lui permet de s’adapter au contexte et de le comprendre pour réaliser sa mission.

\subsubsection{La figure du collègue}

Le référent d’activité joue également un rôle dans l'intégration du bénévole dans l'équipe :

Et donc, intégrer le bénévole dans léquipe, et ben ça fait partie aussi de l'acte professionnel ${ }^{8}$.

Les réunions, les temps conviviaux sont identifiés comme des occasions d’associer le bénévole à la vie de la structure, que ce soit dans les réunions avec les professionnels (en lien avec l'activité) ou bien le partage de moments festifs (fêtes de quartier ou sorties). Dans le centre 2, les temps de réunion permettent d'identifier un ressenti collectif vis-à-vis des actions menées, d'en repérer les limites et les points d'amélioration. Ces moments sont des occasions pour chaque bénévole de s’exprimer, de faire des critiques; dans un objectif de construction partagée.

Dans ces accompagnements, on retrouve la figure du collègue qui correspond à ce que Wittorski (2007) appelle la voie de professionnalisation $\mathrm{n}^{\circ} 1:$ « la logique de l'action» (p. 114) ou logique d’apprentissage sur le tas. Le bénévole ainsi accueilli se retrouve "dans des situations dites d'improvisation... intelligence du contexte... adaptation immédiate de tout être vivant à son environnement du fait de son interaction forte entre l'individu, son action, l'environnement et ses propriétés » (Ibid.). Le bénévole ajustera son action notamment par le biais des questionnements que pourront provoquer certaines situations.

\subsubsection{Formations techniques en interne; la figure du formateur}

Dans le centre 3, les formations sont plutôt construites en interne : En interne, on fait des formations spécifiques; dailleurs chaque activité a son programme... si on peut faire en interne, on fait en interne ${ }^{9}$.

Le centre 2 a eu l'occasion d'expérimenter plusieurs types de formation, notamment dans le cadre de laccompagnement scolaire.

Entretien Directeur centre 1.

Entretien Référent accueil centre 3. 
Certaines formations hyper théoriques (voie $\mathrm{n}^{\circ} 6$; culture de l'enseignement, formation magistrale) navaient présenté : aucun intérêt pour les bénévoles; ils sont partis en disant que cétait intéressant dans l'absolu.

Plus récemment, la mise en place d'une formation pratique a permis aux bénévoles de repartir avec des outils visant à améliorer leur action On a besoin de savoir comment travailler avec un jeune qui refuse d'amener ses cahiers. Voilà c'est des choses pratico-pratiques.

Ces formations à visée technique se rapprochent de la voie de professionnalisation $\mathrm{n}^{\circ} 2:$ "logique de la réflexion et de l'action " (Wittorski, 2007, p. 116) qui permet d'inscrire l'activité dans sa dimension collective, par une approche visant le fonctionnement (les outils) et la dimension du travail en équipe : "Recherche de connaissances ou de savoirs utiles pour agir... les individus construisent des connaissances dans l'action " (Ibid.). Pour le bénévole, il s’agit d'un apport de connaissances directement transférable à l'activité, visant la prise de fonction et la compréhension de certains aspects de sa pratique.

\subsubsection{Analyse des pratiques; la figure de l'animateur}

La complexité de certains accompagnements sociaux nécessite parfois un autre regard et un soutien extérieur, permettant de prendre du recul sur les pratiques mises en œuvre: On sést souvent dit que de temps en temps, avoir un psychologue dans l'équipe, ce serait pas mal; pour avoir des outils pour apprendre à gérer des difficultés ${ }^{10}$.

Cette thématique renvoie en formation, à la question de l'analyse des pratiques, qui se développe dans le champ du travail social permettant aux professionnels d'ajuster leurs positionnements et de parfois mieux gérer leurs affects dans le cadre d'accompagnement où l'objectivité est de rigueur. C'est vrai que ça pourrait être intéressant d’avoir un regard extérieur qui puisse parfois aussi, pour l'animateur, le rassurer, sur ce qu’il peut faire, ne pas faire, et de toute façon la limite de ce qu'il va pouvoir faire ${ }^{11}$.

\section{Les formations proposées via la fédération}

Le centre 2 collabore régulièrement avec la fédération parisienne pour faciliter l'accès à la formation des bénévoles, notamment sur la base des propositions du catalogue. L'avantage c'est de rencontrer des acteurs d'autres centres sociaux et déchanger sur les bonnes et les moins bonnes pratiques.

Le centre 2 semble envisager la formation des bénévoles dans sa dimension d’origine qui consiste à former les acteurs pour que chacun puisse prendre sa place et devenir acteur du projet en toute connaissance de cause. Laccès à la formation c'est aussi le moyen de former des citoyens qui prennent part à un projet local, de quartier. C'est important qu'ils aient un minimum de bagages et puis surtout qu'ils comprennent l'idée dans laquelle ça se fait dans les centres sociaux... ${ }^{12}$.

Lanalyse des pratiques s'inscrit pour Wittorski (2007) dans les voies de professionnalisation $\mathrm{n}^{\circ} 3$ et $4:$ : logiques de la réflexion sur et pour laction » (p. 116-117). Elle se déroule dans les organisations qui instituent des moments de formalisation des pratiques soit de façon rétrospective (voie $\left.\mathrm{n}^{\circ} 3\right)$ soit par anticipation (voie $\left.\mathrm{n}^{\circ} 4\right)$.

\subsection{Pratiques-dispositifs de professionnalisation? Développement personnel et professionnel}

Le repérage des cultures de formation en centre social a permis d'identifier quatre catégories permettant de formuler des caractéristiques pour chacune d'elles. Il sest ensuite avéré nécessaire de différencier pour chacune des voies de professionnalisation, celles qui relevaient de dispositifs formels, de celles qui s’apparentaient davantage à des pratiques informelles; cette distinction permettant de mettre en évidence la place de l'individu dans les logiques institutionnelles de formation (Wittorski, 2005).

La voie de professionnalisation $n^{\circ} 1$ qui correspond à la formation sur le tas (recherche de participation au projet global de lassociation, réunions) est présente dans les trois centres où la figure du collègue apparaît sous une approche individuelle ou collective, favorisant la transmission des valeurs et l'intégration dans l'équipe. Elle est particulièrement préconisée dans le centre 2 qui développe en parallèle des occasions permettant aux bénévoles de prendre la parole et de donner leur avis dans les instances de concertation. Pour Wittorski (2007), cette voie de professionnalisation n'implique pas de dispositifs particuliers; elle s'appuie sur des pratiques informelles visant la mobilisation de compétences efficaces pour la situation.

La voie de professionnalisation $n^{\circ} 2$ qui regroupe les formations techniques proposées en interne (centre 3 notamment) s'inscrit dans une logique de professionnalisation liée à l’activité impliquant le travail d’équipe (compétences collectives). En effet, dans cette voie, Wittorski distingue les dispositifs (formalisation de la formation par exemple dans le cadre de l'alternance) des pratiques (sollicitation des collègues). Or dans le centre 3, les formations dispensées en interne (proximité entretenue avec le formateur qui est aussi un collègue) sont animées par les salariés de l'association, relevant alors plus d'une pratique (mobilisation de connaissances directement utiles pour l'action) que d'un

Entretien Directeur centre 2.

11 Ibid 13.

12 Ibid 13. 
dispositif (où les savoirs permettent d'intellectualiser les compétences).

Les voies de professionnalisation 3 et 4 qui accompagnent l'analyse des pratiques sont présentes dans le centre 2 via les formations de la fédération ou celles proposées en interne (animateur extérieur déployant des contenus de formation basés sur un cahier des charges préalablement établi en fonction des besoins). Pour Wittorski (2007), il s'agit majoritairement de dispositifs qui visent d'abord une professionnalisation des activités (voire de lorganisation) par une formalisation des pratiques; mais ces logiques peuvent aussi permettre aux individus délaborer des connaissances sur leur action. Ainsi ces voies de professionnalisation sont des dispositifs qui peuvent « servir des enjeux séparés ou combinés de professionnalisation des activités ou de l'organisation, mais aussi de l'individu.

La voie de professionnalisation $n^{\circ} 5$ est développée dans chaque centre, plus particulièrement dans les centres 1 et 3 . Ces démarches de tutorat visent la transmission des savoirs et connaissances utiles pour contribuer à une représentation collective de la situation. Il n’y a pas vraiment de distinction entre pratiques et dispositifs, mais dans les structures rencontrées, ce tutorat semble plutôt relever d'une pratique en lien avec la culture du centre social (Wittorski, 2005) : « Le savoir qu'il soit théorique ou d'action guide les dispositifs de professionnalisation, alors que laction et les compétences guident les individus dans leurs pratiques de professionnalisation... elles ont pour préoccupation dominante l'action et l'efficacité de cette action» (p. 205).

\section{De quelle professionnalisation parle-t-on?}

Pour Wittorski (2007), la professionnalisation relève d'une intention sociale qui porte une charge idéologique forte. Cette approche correspond particulièrement bien au contexte associatif aujourd'hui : dans un objectif d'efficacité, cette logique institutionnelle correspond à l'idée d'une professionnalisation-efficacité (logique des organisations) qui vise à flexibiliser les salariés ${ }^{13}$ et qui influence les parcours et pratiques bénévoles.

Les bénévoles inscrivent leurs pratiques dans des activités professionnalisées et agissent avec professionnalisme par la mise en œuvre de compétences attendues; quant aux objectifs de qualification sous-jacents, ils dépendront de la volonté associative de participer au développement professionnel des bénévoles. Ainsi se distingue la professionnalisation des structures (enjeux institutionnels), des activités (développement des pratiques professionnelles) et des individus (développement professionnel/personnel).

Dans ce triptyque qui articule trois niveaux de logiques et d'enjeux différents, nous retrouvons les dimensions de la professionnalisation en centre social : La professionnalisation de l'organisation proprement dite; cette dimension est bien visible, notamment par l'intégration de pratiques professionnelles et le développement des logiques de management. Elle pose la question des modes de gouvernance et des enjeux participatifs dans un secteur où l'on vise la promotion sociale (éducation populaire). La professionnalisation des activités (qui se distingue notamment par l'appropriation d'outils et le respect des règles du métier) semble se développer, mais la formalisation est-elle suffisante permettant d’en identifier les règles de fonctionnement (répartition salariés/bénévoles dans les activités « centre social ») ? La professionnalisation des bénévoles reste la plus difficile à identifier. Les bénévoles inscrivent leurs pratiques dans des activités professionnalisées et agissent avec professionnalisme par la mise en œuvre de compétences attendues. Si la notion d'efficacité est recherchée de façon prioritaire pour que le bénévole puisse mettre en œuvre les compétences adaptées à la mission qui lui a été confiée, la quête de reconnaissance personnelle est-elle assouvie?

Il est ressorti des entretiens des logiques de professionnalisation différentes d'un centre à l'autre. Elles s'inscrivent toutes, à des degrés divers, dans les dimensions identifiées par Wittorski (2007) : organisation, activité, individu, avec le dénominateur commun activité qui se décline selon 3 modalités :

\subsubsection{Modalité 1 : Professionnalisation Activité/organisation}

Le centre 1 fait l'objet d'une professionnalisation globale de la structure (peu de marge de manœuvre quand aux procédures de recrutement et aux niveaux de diplômes requis). Si la professionnalisation est acquise, tant du côté de lorganisation que des activités, elle est d’abord envisagée du côté des professionnels; mais les enjeux liés à la participation montrent aussi une volonté de développer des dispositifs souples à destination des bénévoles leur permettant de s’intégrer dans la structure.

\subsubsection{Modalité 2: Professionnalisation Activité/individu}

Dans le centre 2, la professionnalisation se développe du côté des exigences de qualification, mais lorganisation globale du centre reste marquée par une volonté de préserver la liberté associative, notamment par le recrutement de personnes non qualifiées, mais disposant d'expériences acquises dans d'autres contextes, notamment militantes. Dans ce centre, l'accès à la formation des bénévoles est rattaché à la professionnalisation, mais constitue aussi le moyen de développer des projets plus personnels (voies 3 et 4 ).

\subsubsection{Modalité 3 : Professionnalisation Activité/qualification}

Dans le centre 3, les contraintes liées à la qualification sont très importantes; le développement de formations en interne montre l'enjeu de répondre aux exigences de professionnalisation, tant du côté des salariés que des bénévoles. Il s'agit donc d'abord d'une professionnalisation

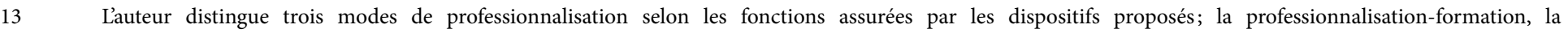
professionnalisation-profession et enfin la professionnalisation-efficacité au travail retenue à ce stade de l'exploration. 
de l'activité qui s’inscrit dans une démarche soumise au contrôle des financeurs.

Les pratiques, en se centrant sur l'action, articulent d'abord l'individu et les compétences utiles; c'est ce qui semble émerger des différents entretiens. Il ressort ainsi une logique récurrente de professionnalisation des activités qui nécessite dengager une analyse des enjeux sociaux qui en découlent alors que les structures valorisent leur appartenance à des valeurs de partage et mutualisation des savoirs (éducation populaire).

\section{Analyse des enjeux sociaux selon les dimensions de professionnalisation visée dans une démarche d’éducation populaire}

\subsection{Les logiques institutionnelles de professionnalisation}

Dans les trois centres, la professionnalisation se fait globalement dans le sens d'un professionnalisme sur la base de compétences acquises ou recherchées par la formation. La notion d'efficacité du côté de l'organisation s'inscrit dans une démarche de rigueur et de responsabilité liée au financement d'un service public (ou par délégation dans le cadre d'un appel d'offres). Les centres sociaux élaborent ainsi des stratégies institutionnelles pour conserver léquilibre des valeurs dans un contexte où la professionnalisation vise d'abord l'efficacité du travail. Il semble qu'une professionnalisation des activités soit recherchée, alors même qu'il n'existe pas d'enjeux de reconnaissance spécifique à un groupe professionnel donné. La professionnalisation des activités, sans forcément répondre à un objectif de constitution d'une profession, engage alors les structures dans une réflexion pour rendre lisible/faire reconnaitre les pratiques. Cela peut notamment s'inscrire dans le développement d'un intérêt pour construire des valeurs communes autour d'une activité donnée. Ainsi la traduction des valeurs en fiches de poste guidant les missions (à l'instar des centres 1 et 3) apparaît comme une volonté de formaliser des outils servant de support aux compétences bénévoles attendues.

Du côté des enjeux pour la formation, les pratiques/dispositifs développés par les centres sociaux s'organisent d'abord en fonction des contextes spécifiques dans lesquels ils sont déployés. Ainsi, des dimensions complémentaires de professionnalisation sont apparues dans chaque centre social, donnant à voir des modalités d'accompagnement différentes vis-à-vis des bénévoles. Si la professionnalité des activités apparaît récurrente, le tutorat individuel semble lui répondre dans une logique de formations en situation de travail évitant toute déperdition dans la transmission des informations. Concernant les formations de la fédération, il est à noter que leur accessibilité par les bénévoles requiert au préalable l’avis du référent d’activité. Parfois, les référents préfèreront se former d’abord sur leur activité avant d’assurer, dans un deuxième temps, la transmission des informations auprès des bénévoles. Le développement de formations en interne (au près des réalités territoriales de la structure) permet davantage d'ajuster les compétences à l'environnement de travail immédiat. Ces pratiques déployées en contexte associatif semblent se rapprocher des dispositifs d'accompagnement au sein des formations intégrées au travail (accueil et intégration des nouveaux salariés) qui permettent aux institutions de s'ajuster aux demandes d'efficacité (nécessité de rendre flexibles les situations de travail). La logique du résultat qui encadre le rapprochement des individus de leurs situations de travail (compétences utiles) tend à articuler davantage la formation aux contextes professionnels (employabilité, compétitivité, flexibilité) permettant d'éviter la juxtaposition des apprentissages (et leur déperdition).

\subsection{Les logiques individuelles de professionnalisation dans l'émergence du tutorat}

Le seul besoin de lentreprise commanditaire (intention sociale) ne saurait être considéré sans prendre en compte les logiques individuelles de professionnalisation associées aux enjeux de développement personnel/professionnel (la professionnalité implicite, notamment). Or, du côté des bénévoles, l'objectif de professionnalisation n’est pas forcément exprimé : il n’en reste pas moins que l'attente de reconnaissance (notamment par le biais du soutien apporté par le référent) reste très forte. Les transmissions réalisées dans le cadre du tutorat interrogent alors l'exercice de cette fonction en émergence dans un contexte associatif en mutation. L'activité tutorale déployée à l'égard des bénévoles est en effet significative d'un contexte social et associatif traversé par des mutations importantes (notamment dans le cadre de la Révision générale des politiques publiques). Pensées à l'origine dans une logique de coopération/mutualisation (éducation populaire), les formations visent aujourd'hui l'efficacité dans la prise en charge des publics. En rapprochant les bénévoles des situations de travail (activités), l'activité du tuteur est alors en tension entre deux formes de logiques : d'un côté, lévolution des formes d'engagement (plus individualiste/pragmatique et parfois avec des attentes d'insertion professionnelle et/ou de lien social) et de l'autre, lévolution des exigences/formes de professionnalisation (qualification des acteurs) dans un contexte defficacité au travail (on attend des bénévoles des compétences).

Le tuteur est donc au centre des dispositifs proposés, entre les projets de la structure dont il se fait le garant à travers ce qu'il transmet au bénévole; et les projets des bénévoles auxquels il doit constamment s’adapter pour respecter leur engagement (identifier leurs besoins individuels, notamment en termes de socialisation). De leur côté, les bénévoles formulent des attentes vis-à-vis des salariés pour leur permettre de réaliser leurs missions et se sentir reconnus dans des activités pour lesquelles ils se mobilisent. Ainsi, lacceptation, par les bénévoles, des formules d'accompagnement proposées, engage aussi les centres sociaux à se positionner sur des modes de coopération adaptés; favorisant d'une part la reconnaissance du travail bénévole et d’autre part le développement d’un engagement individuel et personnel. 


\section{Conclusion}

Dans un contexte de transmissions professionnelles réalisées dans le respect des valeurs déducation populaire, telles que la mutualisation ou le partage des connaissances, la recherche de compatibilité, entre les logiques individuelles et institutionnelles, semble alors essentielle au bon fonctionnement des situations de travail dans lesquels les bénévoles s'impliquent. La connaissance des profils d’engagement permet alors de favoriser l'ajustement des attentes bénévoles (professionnelles, sociales, personnelles) aux logiques de l'organisation (implication dans la structure, lien social, insertion professionnelle). Au-delà du contre don attendu, le recueil des attentes et projets visés par les bénévoles (dans l'exercice de leur bénévolat) apparaît comme une donnée préalable au bon déroulement de l'activité. Cette démarche de gestion des ressources/compétences semble par ailleurs indispensable à la préservation des valeurs du projet associatif.

\section{Références bibliographiques}

Lesain-Delabarre, J.M. (2006). Les valeurs dans l'action sociale. Dans J.-Y. Barreyre \& B. Bouquet, Nouveau dictionnaire critique d'action sociale (p. 619). Paris : Bayard.

Tchernonog V. (2008). Les grandes évolutions du secteur associatif français. Recma, (309), 11-26.

Wittorski R. (2005). Les dynamiques de professionnalisation des individus, des activités et des organisations. Dans M. Sorel \& R. Wittorski (dir.), La professionnalisation en actes et en questions (p. 211-243). Paris : L'Harmattan.

Wittorski R. (2007). Professionnalisation et développement professionnel Paris : L'Harmattan. 